\title{
Le cyberharcèlement à l'école : État des lieux et perspectives éducatives
}

\section{Educational Responses to Cyberbullying in Schools}

\section{Ciberacoso en la escuela: situación actual y perspectivas educativas}

Bérengère Stassin, maître de conférences en sciences de l'information et de la communication Laboratoire Crem, Université de Lorraine, France

berengere.stassin@univ-lorraine.fr

RÉSUMÉ

Avec l'arrivée des smartphones et des réseaux sociaux au milieu des années 2000, une nouvelle forme de violence et de harcèlement scolaires a fait son apparition : la cyberviolence et le cyberharcèlement. Depuis la loi du 8 juillet 2013 pour la refondation de l'école de la République, la lutte contre « toutes les formes de harcèlement » est devenue une priorité. Cet article présente les différentes formes de cyberviolences exercées entre élèves, les caractéristiques du cyberharcèlement à l'école et les principales actions de prévention mises en place, en France, depuis le début des années 2010. L'article propose ensuite de montrer en quoi l'éducation aux médias et à l'information, l'éducation à l'esprit critique, l'éducation à l'empathie et le développement des compétences émotionnelles sont des armes efficaces pour lutter contre le phénomène.

Mots-clés : compétences émotionnelles, cyberharcèlement, cyberviolence, éducation à l'empathie, éducation à l'esprit critique, éducation aux médias et à l'information, harcèlement scolaire.

ABSTRACT

The advent of smartphones and social networks in the mid-2000s has brought with it new forms of school violence and bullying: online violence and cyberbullying. Since the French law of 8 July 2013, aimed at reforming the school system, the fight against "all forms of bullying" has become a priority. This article will analyze the different forms of online violence amongst 
students, it will illustrate the characteristics of cyberbullying at school and it will then present the main prevention measures put in place in France since the beginning of 2010. Finally, it intends to show how Media and Information Literacy, Empathy Education and the development of both students' critical thinking and their emotional skills are effective weapons in the fight against the phenomenon.

Keywords: critical thinking, cyberbullying, emotional skills, empathy education, media and information literacy, online violence, school bullying

\section{RESUMEN}

Con la llegada de los teléfonos inteligentes y de las redes sociales alrededor del año 2005, surgió una nueva forma de violencia y de acoso escolar: la ciberviolencia y el ciberacoso. La ley para la refundación de la escuela de la República y la lucha contra "todas las formas de acoso", promulgada el 8 de julio de 2013, se ha convertido en una prioridad. En este artículo presentaremos, para empezar, las diferentes formas de ciberviolencia ejercidas entre los estudiantes, las características de la ciberdelincuencia en la escuela y las principales acciones de prevención implementadas en Francia desde principios del año 2010. Después, mostraremos que la educación en medios, la información, la educación del pensamiento crítico, la educación de la empatía y el desarrollo de habilidades emocionales son armas efectivas para combatir este fenómeno

Palabras clave: habilidades emocionales, ciberacoso, ciberviolencia, educación de la empatía, pensamiento crítico, educación en medios de comunicación, acoso escolar

En France, environ $10 \%$ des élèves sont victimes chaque année de harcèlement scolaire, c'est-à-dire de violences qui sont exercées de manière répétée à leur encontre et qui ne sont pas sans conséquence sur leur santé et leur scolarité. S'il y a quelques années encore, les élèves harcelés trouvaient un peu de répit une fois sortis de l'école, il n'est pas rare aujourd'hui qu'on les poursuive en dehors des temps et des lieux scolaires, par le biais des smartphones et des réseaux sociaux. La violence devient cyberviolence et le harcèlement, cyberharcèlement. Mais ces outils sont aussi utilisés dans l'enceinte de l'école pour se moquer d'un camarade, l'insulter, le photographier à son insu. La récente " interdiction du téléphone portable » est d'ailleurs censée limiter, en autres, la diffusion de contenus violents et choquants au sein des établissements. La cyberviolence est désormais une réalité et $20 \%$ des élèves en ont déjà été victimes (Blaya, 2013). Tantôt elle prolonge " en ligne " un harcèlement qui a déjà lieu " hors ligne », tantôt elle émerge sur les réseaux sociaux (p. ex. une photo compromettante est publiée un samedi soir) et déclenche une vague de brimades en face à face. Comment l'école peut-elle faire face à l'intrusion de la cyberviolence en son sein? Quelles sont les perspectives éducatives ouvertes par cette intrusion? Le problème du cyberharcèlement peut-il être traité indépendamment de celui du harcèlement scolaire?

\section{Vers une définition du cyberharcèlement}

Un élève est victime de harcèlement lorsque des violences sont exercées de manière répétée à son encontre et dans l'intention de le blesser et qu'elles sont inscrites dans une relation asymétrique (son agresseur exerce une domination physique et/ou psychologique sur lui ou bien le malmène avec la 
complicité d'un groupe de pairs dont il est le meneur). Par analogie, on peut dire qu'il y a cyberharcèlement lorsqu'un élève est soumis à des cyberviolences répétées et visant à lui nuire, dans un contexte où il y a déséquilibre des forces (il est insulté par plusieurs camarades, ou bien reçoit des messages anonymes et ne sait pas qui les envoie). Cependant, la question de la « répétition » est plus difficilement appréhendable en contexte numérique. Les logiques de fonctionnement propres au web peuvent conférer une dimension harcelante à une attaque unique et ponctuelle.

Commençons par présenter les différentes formes de cyberviolence entre pairs. On distingue tout d'abord l'exclusion sociale (un élève est exclu ou banni d'un groupe sur Facebook ou Snapchat) et l'usurpation d'identité (un faux profil est créé à son nom et des contenus compromettants y sont publiés). La cyberviolence peut également être verbale : surnoms méchants, insultes en lien avec l'origine, la religion, la tenue vestimentaire, l'apparence physique ou ancrées dans le sexisme et l'homophobie (DEEP, 2014; Couchot-Schiex et Moignard, 2016). Elle peut être physique et consister à filmer des agressions (les Anglosaxons parlent ici de happy slapping) ou à perpétrer une agression en vue de la filmer et de la partager sur les réseaux sociaux (Tisseron, 2011). Enfin, elle peut être sexuelle : envoi d'images érotiques ou pornographiques dérangeantes, voire choquantes, pour le destinataire; publication de photos ou vidéos « intimes » sans le consentement de la personne qu'elles représentent à des fins de vengeance (revenge porn) suite à une rupture sentimentale, à une dispute, un différend ou par jalousie; publication de photos prises sous la jupe d'une fille (upskirting) ou de photos volées d'un décolleté trop plongeant (creepshot).

Des cyberviolences répétées sont bien du cyberharcèlement, mais un contenu "violent » posté une fois par une personne peut aussi se transformer en cyberharcèlement, et ce, par les likes, partages et commentaires dont il peut faire l'objet. Ces « approbations » lui confèrent un caractère répétitif : "Liker, c'est déjà harceler ", scandait le slogan de la deuxième journée de mobilisation nationale contre le harcèlement scolaire (3 novembre 2016). La répétition peut également venir de la pérennité des traces numériques : " une insulte virtuelle a le potentiel de rester définitivement » (Boudreault, 2014, p. 29). Les photos intimes ou vidéos humiliantes sont généralement supprimées des sites sources, mais leur copie et leur partage font qu'elles sont toujours stockées quelque part. Soumises à l'« intelligence des traces " (Merzeau, 2013), elles peuvent ressurgir à tout moment, des mois, voire des années plus tard, entachant à nouveau l'image, la réputation et l'identité numérique de la victime. Cette identité se définit en effet comme l'ensemble des traces laissées volontairement ou non, consciemment ou non, par nos activités numériques, ou que les autres laissent de nous lorsqu'ils nous identifient, par exemple, dans une publication. Insulter une fois un camarade en ligne peut donc avoir des conséquences nettement plus importantes que de l'insulter une fois en face à face.

\section{Le cyberharcèlement à l'école}

Le climat scolaire et l'atmosphère générale qui règnent au sein d'une classe ou d'un établissement influencent nécessairement la qualité des relations entre élèves. Une classe qui se caractérise par un mauvais climat a plus de chance de voir émerger en son sein des situations de harcèlement et de cyberharcèlement. La cyberviolence et le cyberharcèlement sont avant tout des violences de proximité, exercées par des groupes sociaux préexistants " hors ligne » et assez souvent par des camarades de

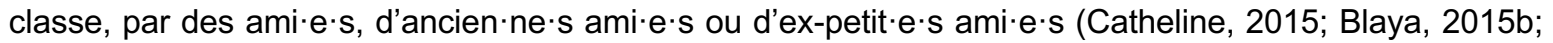
Felmlee et Faris, 2016; Boyd, 2016).

Bien que la continuité entre harcèlement scolaire et cyberharcèlement ne soit pas toujours avérée, les agresseurs et les victimes sont tout de même souvent impliqués " hors ligne " et " en ligne ", dans 30 à $70 \%$ des cas, selon les études : des violences sont exercées en face à face au sein de l'établissement et se poursuivent sur les réseaux sociaux ou bien une violence est perpétrée sur les réseaux sociaux et 
déclenche une vague de brimades infligées en face à face (Blaya, 2015a). Parmi les cyberagresseurs se trouvent donc des élèves qui sont déjà impliqués dans des actes de violence hors ligne et parmi les cybervictimes, se trouvent des élèves qui sont déjà victimes hors ligne. On retrouve aussi les suiveurs qui participent aux méfaits en likant, partageant ou commentant les contenus, ainsi que les témoins passifs, qui les lisent, mais ne « disent » rien.

En contexte numérique, d'autres parties prenantes font leur apparition, rendant l'analyse du phénomène plus complexe (Bellon et Gardette, 2013) : ceux qui font l'objet de moqueries voire de harcèlement hors ligne et qui se servent de leurs compétences informatiques pour se venger de leurs harceleurs; ceux qui ne font pas l'objet de moqueries et encore moins de harcèlement, mais qui se servent de leurs compétences informatiques pour venger les victimes et punir les harceleurs; ceux qui se métamorphosent et se désinhibent derrière leur écran et se livrent à des actions qu'ils s'interdiraient totalement hors ligne; ceux qui publient ou relaient un contenu par mégarde, par inadvertance, ou sur le feu de l'action, sans prendre le temps de réfléchir aux conséquences de leur « clic ». À ces quatre catégories s'ajoutent bien sûr les personnes totalement extérieures à l'établissement scolaire des victimes et agresseurs, mais « amis » avec eux sur les réseaux sociaux.

Les filles sont deux fois plus victimes que les garçons (Couchot-Schiex et Moignard, 2016); les homosexuels et les transsexuels risquent quatre fois plus d'endurer un épisode de cyberharcèlement (Felmlee et Faris, 2016). Les garçons non homosexuels, mais ne répondant pas aux normes de genre, peuvent aussi être victimes d'homophobie du fait d'une pression viriliste qui peut régner au sein du groupe de pairs (Debarbieux et al., 2018). Concernant le genre des cyberagresseurs, Catherine Blaya (2015a) souligne des contradictions dans la littérature scientifique : des auteurs mettent en avant que les filles sont plus impliquées que les garçons, qu'elles ont tendance à s'en prendre à d'autres filles appartenant à leur propre réseau amical; d'autres, au contraire, montrent que les garçons s'adonnent plus que les filles à la cyberviolence; d'autres encore montrent que les filles et les garçons sont autant impliqués, mais que les premières seraient plus engagées dans la profération d'insultes et de moqueries et dans le lancement de rumeurs alors que les seconds seraient plus enclins à diffuser des photographies humiliantes et des photographies à caractère sexuel représentant des filles.

Soulignons que la cyberviolence frappe aussi les enseignants, bien que peu d'études soient consacrées à ce phénomène : des photos volées pendant les cours, publiées et commentées sur Snapchat, des rumeurs et des insultes qui circulent à leur encontre en ligne, au sein de groupe fermé ou de manière publique; des enseignantes qui voient leur entrejambe photographié par un élève qui introduit son téléphone portable sous leur jupe ${ }^{1}$. Au Québec, le nombre d'enseignants victimes de cyberviolence se chiffre à 5,6\% (Villeneuve et al., 2016). Pour certains, les actes - qui peuvent être perpétrés par des élèves, des parents ou encore des collègues - sont ponctuels. Pour d'autres, ils s'étendent sur plusieurs mois, constituant bien une forme de cyberharcèlement. II n'existe pas, en France, d'étude similaire, mais différents cas sont rapportés chaque année aux Autonomes de solidarité laïque, des associations départementales qui œuvrent pour la défense des personnels enseignants.

\footnotetext{
${ }^{1}$ On peut ici citer en exemple le cas d'Hélène, professeure d'anglais âgée de 47 ans, qui a découvert en novembre 2018 qu'une vidéo et une série de photos prises sous sa jupe ou insistant sur ses parties intimes (et parfois accompagnées de gestes et de propos obscènes) circulaient sur les réseaux sociaux. Source : https://www.radiototem.fr/tarn-et-garonne/4391-caussade-l-enseignante-filmee-sous-sa-jupe-ason-insu-temoigne
} 


\section{Une prise de conscience récente}

Le harcèlement scolaire est longtemps resté en France englobé dans les débats sur la violence scolaire en général. Ce n'est qu'au début des années 2010, suite aux enquêtes de victimation réalisées par Éric Debarbieux (2011a, 2011b) et révélant que $10 \%$ des élèves sont victimes de violences répétées, que les pouvoirs publics prennent conscience de l'ampleur du phénomène.

Différentes actions sont dès lors mises en place : organisation des premières assises sur la prévention du harcèlement en mai 2011; lancement de la première campagne d'information et mise en place d'une ligne d'écoute téléphonique nationale et gratuite (3020) en 2012; création d'un site Internet ministériel proposant des ressources aux enseignants et aux parents; lancement du concours académique "Non au harcèlement » en 2013.

L'année 2013 est également marquée par le suicide, le 13 février, de la jeune Marion Fraisse, 13 ans, victime de harcèlement scolaire, mais aussi de cyberharcèlement. Cette tragique histoire, en plus de réaffirmer la nécessité d'agir, met en exergue que harcèlement et cyberharcèlement peuvent constituer les deux faces d'une même pièce, que le harcèlement sort de l'école par le biais des réseaux sociaux pour mieux y revenir, et que ce sont deux fléaux qui doivent être traités et combattus indistinctement.

La lutte " contre toutes les formes de harcèlement » se retrouve donc inscrite dans la loi $n^{\circ} 2013-595$ du 8 juillet 2013 d'orientation et de programmation pour la refondation de l'École de la République : « [elle] sera une priorité pour chaque établissement d'enseignement scolaire. Elle fera l'objet d'un programme d'actions élaboré avec l'ensemble de la communauté éducative ".

En 2015, le dispositif « Ambassadeurs Lycéens », plaçant les élèves au cœur des actions de prévention, est créé et une journée de mobilisation nationale est instaurée. Sa quatrième édition s'est tenue le jeudi 8 novembre 2018 et avait pour slogan «Une photo c'est perso, la partager c'est harceler ». Elle sensibilisait, cette année, au revenge porn et à la diffusion non consentie de photos intimes.

Différentes actions de prévention peuvent bien sûr être réalisées par les établissements scolaires et plus particulièrement par le Comité d'éducation à la santé et à la citoyenneté (CESC). Ce dernier peut s'appuyer sur différents partenaires extérieurs, comme les associations spécialisées dans la lutte contre le harcèlement scolaire (p. ex. Marion La Main Tendue, You Are Heroes) ou dans la lutte contre les cyberviolences (p. ex. e-Enfance, Respect Zone), la police et la gendarmerie, les travailleurs sociaux, les parents. Cependant, la prévention ne peut à elle seule endiguer le phénomène, car il ne suffit pas d'être informé d'un risque pour l'éviter ou de dénoncer un comportement pour qu'il soit abandonné.

\section{L'éducation aux médias et à l'information (EMI) et l'éducation à l'esprit critique}

Inscrite dans la loi de refondation de l'école du 8 juillet 2013, l'EMI vise à « permettre aux élèves d'exercer leur citoyenneté dans une société de l'information et de la communication », à « former des "cybercitoyens" actifs, éclairés et responsables de demain $\aleph^{2}$. Elle a donc pour objectif d'éveiller l'esprit critique des élèves, de leur apprendre à exercer une citoyenneté informationnelle et numérique, de les sensibiliser à la pluralité des sources d'information et à la complexité actuelle de leur univers informationnel et communicationnel. Pour mettre en place cette éducation, les enseignants peuvent s'appuyer sur différents documents :

\footnotetext{
${ }^{2}$ Source : https://eduscol.education.fr/cid72525/presentation-de-I-emi.html
} 
le dossier EMI sur Eduscol', la matrice EMI proposée par le groupe TraAM Documentation de l'académie de Toulouse ${ }^{4}$. Cette matrice propose six grands objectifs, organisés en quatre facettes (Information documentation, Éducation aux médias, Culture numérique, Citoyenneté et éthique) ainsi qu'une progression du cycle 3 au lycée. Ils peuvent également s'appuyer sur les dispositifs tels que les EPI ${ }^{5}$ (Information, communication, citoyenneté), sur les différents parcours, notamment le Parcours citoyen ${ }^{6}$, mais aussi sur la plateforme $\mathrm{PIX}^{7}$ qui permet d'évaluer les compétences numériques des élèves, de la $4^{\mathrm{e}}$ au lycée.

Lorsque l'on évoque les "médias » et l'« information », on pense souvent aux médias de masse et à l'information journalistique, ou encore à la lutte contre la désinformation circulant au sein des médias sociaux (réseaux, blogs, sites, wikis) qui sont devenus aujourd'hui des sources à part entière d'information pour les adolescents, mais des sources dont la fiabilité n'est pas toujours de mise. II y a donc bien nécessité à les former à la recherche d'information, à la validation de l'information, à l'identification des sources et des modes de production de l'information au sein du web (Simonnot, 2007; Serres, 2012) et à les faire passer progressivement d'une posture de consommateur de plateformes à celle d'acteur responsable, de les confronter aux différents supports et sources d'information, de les initier au repérage de la nature des messages (opinion, rumeur, propagande).

Les activités connectées des élèves (recherche ou diffusion de contenus) laissent nécessairement des traces qui révèlent leurs préoccupations et centres d'intérêt et qui sont utilisées par les géants du web à des fins de profilage et de diffusion de publicités ciblées. Une réflexion sur l'apparente gratuité du web et l'utilisation qui est faite de leurs données personnelles est aussi nécessaire. Ces traces sont en outre constitutives de leur identité numérique. Et apprendre aux élèves à maîtriser les différents aspects de leur identité numérique est aussi un enjeu majeur de l'EMI.

Ils doivent être conscients des risques (juridiques) que peuvent leur faire encourir certaines publications qui ne respecteraient pas le droit d'auteur ou le droit à l'image ou qui relèveraient du dénigrement, de la diffamation en ligne, du happy slapping, du revenge porn ou plus globalement du cyberharcèlement. Ils doivent aussi prendre conscience des conséquences que leurs publications peuvent avoir à court, moyen et long terme (Stassin et Simonnot, 2018) :

- les contenus qui apportent la preuve des méfaits en cas de dépôt de plainte;

- les contenus qui ressurgissent des années plus tard et qui entachent à nouveau la réputation des victimes;

- de vieux tweets qui sont déterrés et qui peuvent avoir des répercussions dans la vie professionnelle de leurs auteurs;

- des contenus qui sont publiés par inadvertance ou sans réelle intention de nuire et qui échappent totalement à leurs auteurs qui se retrouvent alors initiateurs d'un cyberharcèlement qu'ils n'avaient pas réellement envisagé;

- des likes accordés un peu trop vite et qui contribuent au lynchage numérique d'une personne.

\footnotetext{
${ }^{3}$ http://eduscol.education.fr/cdi/actualites/archives/dossier-emi-sur-eduscol

${ }^{4}$ Disponible sur : https://disciplines.ac-toulouse.fr/documentation/sites/documentation/files/fichiers/matrice-emi-book.pdf

${ }^{5}$ Enseignements Pratiques Interdisciplinaires

${ }^{6}$ De l'école au lycée, le parcours citoyen vise à aider les élèves à se construire un jugement moral et civique, et à acquérir un esprit critique et une culture de l'engagement.

${ }^{7}$ Pix est un service public en ligne d'évaluation, de développement et de certification des compétences numériques : https://pix.fr/
} 
L'éducation aux médias et à l'information et l'éducation à l'esprit critique qu'elle sous-tend constituent un enjeu éducatif majeur pour les jeunes générations, une arme certes efficace contre les fakes news et la désinformation (Pierre, 2018), mais aussi contre la cyberviolence et le cyberharcèlement. Elle vise, entre autres, à leur apprendre à réfléchir aux contenus qu'ils consultent, publient ou relaient, mais surtout à résister à toute forme d'emprise et à dépasser certains préjugés, certains stéréotypes liés au genre, à l'origine, à l'apparence physique ou à la classe sociale et les discours de haine ancrés dans le sexisme, l'homophobie, le racisme, l'antisémitisme.

L'EMI est bien évidemment un dispositif propice à la valorisation des pratiques numériques des élèves. S'appuyer sur ce qu'ils savent et aiment faire est aussi un levier pour développer leur esprit critique et renforcer leurs compétences numériques. Ces dernières peuvent être mobilisées dans le cadre de la prévention du [cyber]harcèlement. Le concours " Non au harcèlement » peut par exemple être l'occasion de leur faire réaliser une vidéo (un clip informatif, un reportage) et de les impliquer dans toutes les étapes de la réalisation. D'autres productions, dans d'autres cadres, peuvent aussi être réalisées : réalisation de BookTubes à partir d'une sélection d'ouvrages traitant du harcèlement, de la discrimination, du racisme, du handicap ou encore de l'homophobie; réalisation d'une web radio ou de podcasts à l'occasion de la journée de mobilisation nationale contre le harcèlement scolaire ou de la semaine de la presse.

Cependant, il nous semble que le renforcement des compétences numériques n'est pas suffisant pour endiguer la cyberviolence s'il n'est pas associé à un renforcement des compétences émotionnelles, car les émotions jouent un rôle important dans les situations de violence et de harcèlement, hors ligne comme en ligne.

\section{L'éducation à l'empathie et le développement des compétences émotionnelles}

Les compétences émotionnelles sont nécessaires à l'adaptation de l'individu à son environnement, à sa socialisation et au développement de sa sensibilité à l'égard des autres. II existe différents dispositifs ludiques qui peuvent être mobilisés par les éducateurs pour développer ces compétences, et ce, dès l'école primaire : les trois mousquetaires, le jardin des sculptures (Zanna et Jarry, 2018), le jeu des trois figures (Tisseron, 2010), le jeu de plateau Feelings (Fougeret-Linlaud et al., 2016) ou encore le jeu de cartes (Valeurs en main) développé par le collectif You Are Heroes ${ }^{8}$.

Les émotions jouent un rôle prépondérant dans les situations de harcèlement : manque d'empathie, alexithymie, peur des représailles, chaque partie prenante du phénomène est traversée par un état émotionnel particulier (Catheline, 2015). Développer les compétences émotionnelles des élèves, leur apprendre à exprimer leurs émotions et à reconnaître celles des autres, à se mettre à leur place, donc à faire preuve d'empathie est pertinent pour lutter contre le harcèlement, mais aussi le cyberharcèlement. En effet, l'absence de face-à-face propre à la communication électronique et l'impossibilité d'accéder au visage de la personne que l'on attaque, donc à ses émotions, sont des facteurs de diminution de l'empathie et favorisent les passages à l'acte : « Le cyberespace facilite des niveaux d'empathie affective et cognitive peu élevés chez les individus de par l'éloignement de la victime et parfois l'anonymat de l'agresseur qui est ainsi plus susceptible d'adopter des comportements inhibés par ailleurs » (Blaya, 2013, p. 118). Cela peut expliquer pourquoi de jeunes gens au demeurant sans histoire et qui n'ont pas l'habitude de s'engager dans des comportements agressifs cèdent à la cyberviolence, ou encore pourquoi des jeunes qui ne connaissent pas la victime participent à son harcèlement, surenchérissent dans les insultes, jusqu'à parfois contribuer à un lynchage.

\footnotetext{
${ }^{8}$ https://www.youareheroes.org/valeursenmain
} 
Le Parcours éducatif de santé (PES) est un cadre propice à l'éducation à l'empathie, au développement des compétences émotionnelles et à la lutte contre le [cyber]harcèlement. Créé par la loi du 8 juillet 2013, réaffirmé par la loi $n^{\circ} 2016-41$ du 26 janvier 2016 de modernisation de notre système de santé et présent de la maternelle au lycée, il poursuit en effet trois objectifs: (1) développer les dix compétences psychosociales définies par l'OMS'; (2) mettre en place des actions de prévention des conduites addictives et des conduites à risques; (3) promouvoir la protection de la santé, qui passe, entre autres, par la création d'un climat d'établissement favorable à la santé et au bien-être de tous, un climat scolaire serein.

\section{Liste de références}

Bellon, J.-P. et Gardette, B. (2013). Harcèlement et cyberharcèlement à l'école : Une souffrance scolaire 2.0. Issy-les-Moulineaux : ESF Editeur.

Blaya, C. (2013). Les ados dans le cyberespace : Prises de risque et cyberviolence. Bruxelles : De Boeck.

Blaya, C. (2015a). Cyberviolence : état de la question. Dans É. Debarbieux (dir.), L'école face à la violence. Décrire, expliquer, agir. Malakoff : Armand Colin, p. 52-64.

Blaya, C. (2015b). Étude du lien entre cyberviolence et climat scolaire : enquête auprès des collégiens d'lle de France. Les dossiers des sciences de l'éducation, 2015(33), 69-90. https://doi.org/10.4000/dse.815

Boudreault, A. (2014). L'adaptation psychosociale des élèves du secondaire victimes de cyberintimidation. Mémoire. Rimouski, Québec, Université du Québec à Rimouski, Unités départementales des sciences de l'éducation, $169 \mathrm{p}$.

Boyd, D. (2016). C'est compliqué. Les vies numériques des adolescents. Traduit de l'anglais par Hervé Le Crosnier, préface de Sophie Pène. Caen : C\&F Éditions.

Catheline, N. (2015). Le harcèlement scolaire. Paris : PUF.

Couchot-Schiex, S. et Moignard, B. (dir.), Richard, G., Observatoire universitaire international d'éducation et de prevention (2016). Cybersexisme : une étude sociologique dans des établissements scolaires franciliens [Rapport de l'étude commandée par le Centre francilien pour l'égalité femmes-hommes], 84 p. Université Paris Est Créteil : Centre Hubertine Auclert. Repéré à : https://www.centre-hubertineauclert.fr/sites/default/files/fichiers/etude-cybersexisme-web.pdf

Debarbieux, É. (2011a). À l'école, des enfants heureux, enfin presque. [Rapport de l'Observatoire européen de la violence à l'École], 42 p. Repéré à :

https://www.unicef.fr/sites/default/files/userfiles/UNICEF FRANCE violences scolaires mars 2011.p $\underline{\mathrm{df}}$

Debarbieux, É. (2011b). Tirer les leçons de l'enquête nationale de victimation et climat scolaire en collège. [Rapport de l'Observatoire européen de la violence à l'École], 8 p. Repéré à : http://media.education.gouv.fr/file/10 octobre/52/9/Victimation-Tirer-les-lecons-de-l-enquetenationale 197529.pdf

Debarbieux, É., Alessandrin, A., Dagorn, J., Gaillard, O. (2018). Les violences sexistes à l'école. Une oppression viriliste. Rapport de l'Observatoire européen de la violence à l'école. [Rapport de l'Observatoire européen de la violence à l'École, $131 \mathrm{p}$. Repéré à : http://prevenance-asso.fr/wpcontent/uploads/2018/06/Les-violences-sexistes-\%C3\%A0-1\%E2\%80\%99\%C3\%A9cole-uneoppression-viriliste.pdf

Direction de l'évaluation, de la prospective et de la performance (DEEP). (2014). « Un collégien sur cinq concerné par la "cyber-violence" ». Dans Note d'information, 2014(39). Repéré à : https://www.fondation-enfance.org/wp-content/uploads/2016/10/ministereeducation_un_collegien_cing_cyberviolence.pdf

${ }^{9}$ Voir : https://www.lecrips-idf.net/professionnels/dossier-thematique/dossier-CPS/CPS-definitions.htm 
Felmlee, D. et Faris, R. (2016). Toxic Ties : Networks of friendship, dating, and cyber vicitmization. Social Psychology Quarterly, 79(3), 243-262. https://doi.org/10.1177/0190272516656585

Fougeret-Linlaud, V., Catheline, N., Chabaud, F. et Gicquel, L. (2016). Le harcèlement scolaire entre pairs. À propos d'une étude en Vienne visant à évaluer l'apport d'un support ludique mettant en jeu les émotions. Neuropsychiatrie de l'enfance et de l'adolescence, 2016(64), 216-223. https://doi.org/10.1016/i.neurenf.2016.03.007

Merzeau, L. (2013). L'intelligence des traces. Intellectica - La revue de l'Association pour la Recherche sur les sciences de la Cognition (ARCo), Association pour la Recherche sur la Cognition, 1(59), 115-135. Repéré à : https://halshs.archives-ouvertes.fr/halshs-01071211

Pierre, S. (2018). Former à l'esprit critique : une arme efficace contre les fake news. The Conversation. Repéré à : https://theconversation.com/former-a-lesprit-critique-une-arme-efficace-contre-les-fakenews-91438

Serres, A. (2012). Dans le labyrinthe. Caen : C\&F Editions.

Simonnot, B. (2007). Évaluer l'information. DocSI, 44(3), 210-216.

Stassin, B. et Simonnot, B. (2018). Traces numériques et lutte contre la cyberviolence scolaire. Dans Colloque international Réseaux sociaux, traces numériques et communication électronique, IUT du Havre; IDEES Le Havre (CNRS, Université Le Havre Normandie), Juin 2018, Le Havre, France. (p. 309-318).

Tisseron, S. (2010). Les effets de la télévision sur les jeunes enfants : prévention de la violence par le "Jeu des trois figures". Devenir, 22(1), 73-93. https://doi.org/10.3917/dev.101.0073

Tisseron, S. (2011). Blogs, jeunes et vidéos, la responsabilité des adultes. Revue Projet, 2011(320), 55-63. https://doi.org/10.3917/PRO.320.0055

Villeneuve, S., Goyette, N., Duroisin, C., Pinte, J.-P. (2016). Le cyber harcèlement envers le personnel enseignant : apprendre à se cyber protéger pour mieux enseigner. Communication au Congrès AREF 2016, 4-7 juillet 2016, Mons. [billet de blogue]. Repéré à : http://blog.educpros.fr/jean-paulpinte/2016/07/04/le-cyber-harcelement-envers-le-personnel-enseignant-apprendre-a-se-cyberproteger-pour-mieux-enseigner/

Zanna O. et Jarry B. (2018). Cultiver l'empathie à l'école. Paris : Dunod. 